\title{
AFFECTIVITÉ, SENS ET AFFECTS : LES ÉMOTIONS COMME ARTICULATION DE LA VIE BIOLOGIQUE
}

\author{
IAN JAMES \\ University of Cambridge
}

\section{AFFECTIVITY, SENSE AND AFFECTS: EMOTIONS AS THE ARTICULATION OF BIOLOGICAL LIFE}

\begin{abstract}
This article argues that attempts by philosophy to think emotions as embodied is caught between the necessity of thinking them as a subjective first person dimension of experience on the one hand and as an objective biological determination on the other. Philosophy has tended to view these two dimensions, qualitative and quantitative respectively, as in either in a parallelism with each other or alternatively has dispensed with either one or the other. Drawing on Georges Canguilhem's biological thinking of "sense" as a mode of functional relationality that constitutes the being of organisms as such, this article argues that emotions are an extension of a more fundamental affectivity proper to biological life in general. This allows for the qualitative dimension of emotions and the objective knowledge of them in biology to be thought in an ontological continuity with each other. It also allows for a renewed thinking of the fundamental continuity of emotional and affective experience in human and non-human animal life.
\end{abstract}

Keywords: Touch, relation, affection, sense, affects, emotions Mots clés : Toucher, rapport, affection, sens, affects, émotions

Affectivité, sens et affects : c'est à partir de ces trois termes que je propose une réflexion sur les émotions qui sera spéculative ; donc une réflexion qui d'un côté pense à la limite de ce que la conceptualité philosophique peut connaître, divulguer ou bien révéler des émotions, et de l'autre côté, une réflexion qui interprète la vie affective d'une façon expérimentale à partir de la pensée biologique. Et cette pensée spéculative, se situant aux limites et de la philosophie et du savoir biologique, posera la question des émotions dans le contexte d'une interrogation des limites en général : les limites qui séparent l’organisme biologique de son environnement et des autres organismes, les limites qui séparent les êtres humains des animaux, et les limites, autant ontologiques quépistémologiques, qui séparent la philosophie spéculative de la science ou bien la science naturelle des sciences humaines.

Depuis son origine, bien évidemment, la philosophie a interrogé les émotions : on sait que Platon, Aristote, Descartes, Spinoza, Hobbes et Hume en ont tous proposé des théories. Je n'ai pas l'intention, dans le cadre limité de cette intervention, de revisiter ces théo- 
ries ni de parcourir cette histoire philosophique complexe et diverse. Commençons donc plutôt avec une récapitulation de définitions de base. "Affecter ", selon le Petit Robert, veut dire : " Toucher (qqn) par une impression, une action sur l'organisme ou le psychisme ». De cette définition le sens de « l'affectivité » s’ensuit d'une façon conséquente : "l'affectivité » signifie, et je cite, une « aptitude à être affecté de plaisir ou de douleur », ou bien « l'ensemble de la vie affective ». Et donc l'affect, c'est un « état affectif élémentaire », c.a.d. l'expérience d'une impression ou d'une sensation simple, par exemple de plaisir ou de douleur, expérience que nous nommons émotion ou sentiment dès qu'elle devient plus complexe ou riche. Dans toutes ces définitions l'expérience du toucher, et donc du sentir et de la sensation, reste, je dirais, primordiale. L'évidence de ce rôle primordial joué par le toucher dans l'affectivité ou dans l'expérience des affects et des émotions est soulignée non seulement par les définitions du dictionnaire mais aussi par l'usage linguistique habituel. On ressent des émotions, on se dit "touché » par ceci ou cela. Il n'y a donc, bien évidemment, pas d'affect ou d'émotion sans être touché de quelque façon que ce soit, sans un ressentir, un sentir, ou une sensation. Et j'ajouterais qu'il n'y a pas d'affect ou d'émotion sans un sens. L'impression produite sur l'organisme qui constitue cette expérience, le fait d'être affecté par quelque chose aura toujours et sans exception un sens pour l'organisme affecté, même, et peut-être surtout, quand ce sens reste en-deçà de toute possibilité de signification linguistique et en-deçà de toute opération de compréhension ou de cognition dites supérieure ou rationnelle.

Dans ce contexte il n'est pas possible qu'une réflexion philosophique ou spéculative sur des émotions ignore la dimension corporelle, et donc biologique, de la vie affective. Mais ceci pose un problème. D'une part les affects et les émotions font partie des qualia de l'expérience ; ils renvoient à l'aspect phénoménale de notre vécu, auquel on n'accède que par introspection subjective. On sait bien que la philosophie a tendance à interroger les émotions à partir de l'expérience vécue à la première personne. D'autre part la biologie, en tant que science se voulant objective, détermine l'existence de l'organisme dans son statut d'objet de connaissance de l'extérieure et à la troisième personne. Si l'on veut vraiment prendre en compte philosophiquement la dimension corporelle des émotions, c'est-à-dire l'affectivité et donc le toucher et le ressentir qui la constituent primordialement en tant que telle, il faut une pensée qui réunira la perspective phénoménale et qualitative qui se dit à la première personne et la perspective biologique et quantitative qui se dit à la troisième personne. Il nous faudrait une pensée naturaliste qui mettrait la philosophie qui interroge le vécu phénoménal et la science qui détermine l'existence objective dans un certain rapport de continuité.

On ne peut pas parler d'un naturalisme des émotions sans se référer d'abord à Spinoza et à sa définition célèbre des affects vers la fin de la troisième partie de son Éthique. Mais cette définition met en évidence ou bien rend plus complexe le problème du qualitatif et du quantitatif qui vient de se poser. Voici la définition que nous donne Spinoza : «L'Affect qu'on dit une Passion de l'âme est une idée confuse par laquelle l'Esprit affirme une force d'exister de son Corps, plus grande ou moindre d'auparavant, et dont la présence détermine l'Esprit à penser ceci plutôt que cela ${ }^{1}$. Cette formulation est tout à fait cohérente avec une perspective naturaliste dans la mesure où elle ne pose pas de séparation ou de distinction

1 Spinoza, B. (1988) : Éthique. Traduit par Bernard Pautrat. Paris : Seuil, p. 347. 
ontologique entre le corps biologique d'un côté et l'âme ou l'esprit de l'autre. Il existerait une seule et même réalité physique corporelle et spirituelle qui précéderait la distinction corps-esprit, donc Spinoza affirme un monisme psychophysique. Mais cette seule réalité se traduit pour nous en tant que corps et esprit, deux perspectives distinctes qui sont dans un rapport de parallélisme ou d'isomorphisme l'une avec l'autre. L'esprit dans ce contexte n'est donc surtout pas autonome par rapport à « la force d'exister de son Corps » qui est affirmée par l'affect, d'où tout le discours spinoziste sur l'impuissance humaine à réprimer les affects ; c'est la Servitude dont il discute dans la quatrième partie de l'Éthique. D'une part cette définition nous indique que les affects, affirmant des forces d'exister de notre Corps, seraient d'une certaine manière le fond de la vie de l'Esprit et de la conscience humaine, le fond donc de l'expérience phénoménale. L'Esprit est constitué par son idée du corps et on est conscient du corps de par la conscience de ses changements. Donc ce sont des affects qui constituent, à sa base, l'Esprit. D'autre part, il se peut qu'on n'ait pas du tout quitté, dans cette définition spinoziste des affects, le point de vue de la première personne. Dans le contexte du parallélisme psychophysique qui définit les affects comme une idée confuse de l'Esprit affirmant le flux d'une force d'exister corporelle, il est tout à fait possible qu'on reste malgré tout entièrement dans le domaine de l'expérience vécue à la première personne et dans la perspective d'une appréhension qualitative de l'existence : il s'agit toujours des qualia de l'expérience. Donc même si le corps ici est pensé métaphysiquement en toute continuité ontologique avec la conscience et la vie de l'Esprit, il n'est pas pensé phénoménologiquement en continuité avec la perspective du corps biologique déterminé comme objet et à la troisième personne, c'est-à-dire selon le point de vue propre à la connaissance scientifique. Le flux des forces du corps et des affects chez Spinoza, et le conatus dont ce flux est l'expression, n'est pas abordable ou accessible par voie de la science biologique.

Ce problème est légué à la tradition philosophique qui hérite de Spinoza. Je pense ici surtout à Nietzsche et à la théorie nietzschéenne de la force et des affects telle qu'elle est reprise par Gilles Deleuze dans ses écrits des années soixante ${ }^{2}$. Même en reconnaissant que la pensée deleuzienne aspire à être une métaphysique qui serait adéquate à la science moderne, et que les philosophes contemporains, tels par exemple Keith Ansell-Pearson, Manuel DeLanda et John Protevi, ont mis cette pensée deleuzienne en continuité avec la biologie et avec la théorie de la complexité en particulier, je dirais que ce problème persiste ${ }^{3}$. Tous ces penseurs trouvent un parallélisme entre la pensée scientifique et la pensée deleuzienne (la question problématique du parallélisme étant toujours à l'avantscène) mais il n'en reste pas moins que les forces et les affects chez Deleuze, comme chez Spinoza et chez Nietzsche, relèvent toujours d'un vécu immanent ou d'une dimension qualitative de l'existence corporelle et de l'expérience, dimension qui ne se prête pas à une détermination scientifique objective et quantitative.

Si l'on passe de la tradition philosophique européenne à la tradition anglo-américaine telle qu'elle s'est développée pendant le vingtième siècle, le problème se pose d'une autre

2 Deleuze, G. (1962) : Nietzsche et la philosophie. Paris : Presses Universitaires de France ; (1968) : Différence et répétition. Paris : Presses Universitaires de France.

3 Ansell-Pearson, K. (1997) : Viroid Life: Perspectives on Nietzsche and the Transhuman Condition. London : Routledge; (1999) : Germinal Life : The Difference and Repetition of Deleuze. London : Routledge. DeLanda, M. (2002) : Intensive Science and Virtual Philosophy. London : Bloomsbury. Protevi, J. (2013): Life, War, Earth: Deleuze and the Sciences. Minneapolis : University of Minnesota Press. 
manière mais d'une façon non moins irréductible. D'un point de vue général on peut constater que la "philosophy of mind » et la psychologie anglo-américaine tendaient, pendant le vingtième siècle, à ignorer les émotions. Pourtant, vers la fin du siècle, autour de l'an 2000 et jusqu'au présent il y a eu un fort regain d'intérêt par rapport aux théories des émotions en Europe et aux États-Unis. Il existe, par exemple, ce qu’on appelle la théorie des affects, ou bien " affect theory » en anglais, qui réunit une très grande diversité et multiplicité de perspectives tirées de la science cognitive, de la neurologie, de la biologie évolutionniste, mais aussi des sciences humaines. Ce domaine est très vaste et il n'est pas possible d'en faire le tour dans le cadre limité de cet argument. Ce qui importe, c'est le fait, ici souligné et reconnu dans le domaine de l' " affect theory », que toute théorie ou philosophie des affects doit tenir compte de la dimension corporelle et donc biologique des émotions. Mais en même temps la théorie doit également prendre en considération la dimension des affects ou des émotions qui ne se réduit pas à la physiologie. Le caractère multi- et interdisciplinaire de l' " affect theory » répond clairement à cette exigence. Il faudrait pourtant se demander si la difficulté du parallélisme psychophysique identifiée chez Spinoza se pose d'une façon également problématique dans ce contexte.

Quant au naturalisme, et c'est ce qui nous intéresse plus particulièrement ici, la tradition anglo-américaine offre une solution radicale au problème de l'expérience qualitative et sa détermination ou indétermination par la science. On peut tracer la trajectoire de cette tradition à partir de ses racines dans le pragmatisme américain, celui de John Dewey par exemple, et jusqu'à son commencement et son évolution ultérieure dans les philosophies de Wilfred Sellars, Willard van Orman Quine, David Lewis et la neuro-philosophie de Paul et Patricia Churchland. Le trait caractéristique de cette tradition c'est le désir de mettre la philosophie dans un rapport de continuité avec la science naturelle et donc de conférer à la connaissance scientifique l'autorité ultime par rapport à la détermination de la réalité. C’est la science donc qui cautionne en principe, à titre d'autorité exclusive, tout ce qui concerne et l'ontologie et l'épistémologie. Or cette valorisation philosophique de la science engendre une tendance à vouloir éliminer tout ce qui résiste à la détermination empirique ou scientifique. Cette tendance n'est nulle part aussi visible que dans le matérialisme éliminativiste de Paul et Patricia Churchland.

La thèse centrale du matérialisme éliminativiste affirme que toute réalité est physique et que toutes nos idées reçues concernant l'expérience intérieure et qualitative constituent une psychologie naïve qui sera à la longue remplacée par la neuroscience. Les émotions, les souvenirs et jusqu'à la conscience, n'existeraient donc pas en tant que tels. Il nous faudra d'autres catégories plus scientifiques qui désigneront des entités susceptibles dêtre déterminées empiriquement. Voici une citation de Paul Churchland : "S’ils sont à la fin physiques, les qualités intérieures devraient être accessibles sur le plan épistémologique non seulement du point de vue de la première personne ou "subjectif"; ils devraient être accessibles aussi d'un ou de plusieurs points de vue objectifs, par quelque instrument qui pourrait scanner l'activité cérébrale par exemple ${ }^{4}$. Dans cette optique finalement le problème des émotions ne se pose même pas. La catégorie de l'émotion appartiendrait à une psychologie naïve qui serait éliminée tout court en faveur d'une détermination

4 Churchland, P. (1996) : "The Rediscovery of Light », The Journal of Philosophy, 93, 5, pp. 211-228, p. 227. 
d'une activité nerveuse ou neurologique parfaitement mesurable dans des conditions expérimentales. La question de la dimension qualitative des émotions est supprimée, mais au prix de supprimer la dimension qualitative de l'existence en général, c'est-à-dire de la conscience et de l'expérience vécue à la première personne.

Cette position naturaliste extrême n'est évidemment pas suffisante comme une théorie ou une philosophie des affects et des émotions. Car éliminer dans ce contexte ce n'est pas théoriser mais plutôt réprimer. On réprime une dimension fondamentale et irréductible de l'expérience au seul prétexte, très peu philosophique, qu'elle n'est pas accessible à la science objective ou quantitative. Même si le sentir, le ressentir et l'affectivité ne sont pas mesurables ou quantifiables il n'en est pas moins sûr qu'on sent et qu'on ressent et que les affects et les émotions ont une existence et une réalité indéniable. Depuis le début, rappelons-nous, notre tâche ici a été de trouver une pensée qui réunirait la perspective phénoménale et la perspective biologique, les points de vue de la première et de la troisième personne.

Ce qu'il nous faut donc, c'est une autre approche à la biologie, une pensée biologique qui n'est pas aussi brutalement matérialiste et réductrice par rapport à l'existence des organismes vivants. Il nous faut une pensée biologique qui serait plus sensible à la spécificité du vivant et de ses interactions avec son milieu. On trouve les ressources pour une telle pensée non pas dans la tradition naturaliste américaine mais plutôt dans la tradition française de l'épistémologie historique et plus particulièrement dans la philosophie biologique de Georges Canguilhem. Canguilhem, on le sait bien, est reconnu pour son influence sur Michel Foucault, sur le développement du structuralisme français pendant les années soixante et sur la réinterprétation althusserienne de Marx en particulier. Mais il est beaucoup moins reconnu dans la tradition anglo-saxonne de la philosophie de la science. Jean Gayon, professeur en philosophie et histoire des sciences à la Sorbonne, a bien noté que la pensée biologique de Canguilhem n'est pas très unifiée mais plutôt dispersée dans des publications diverses écrites pendant plusieurs décennies, d'où probablement la difficulté de sa réception en dehors de la France. Dans un article sur la question de l'individualité dans la philosophie biologique de Canguilhem Gayon met l'accent sur le caractère relationnel de sa compréhension des organismes vivants. Chez Canguilhem « les individus », souligne Gayon, " ne devraient pas être considérés comme des étants mais plutôt comme des rapports $»^{5}$. Du point de vue biologique, il continue tout en citant Canguilhem directement: " Un vivant est toujours "un système de référence absolu et irréductible". Cette approche revient à dire que l'axiologie s'enracine dans les fondations conceptuelles de la biologie ${ }^{6}$. Cette remarque est à retenir car elle souligne que les notions vagues de matière ou de substance physique, c'est-à-dire les niveaux d'explication auxquels la biologie tend à réduire l'existence et l'activité du vivant, ne sont pas du tout suffisantes pour saisir la spécificité de la vie. Ce qui est bien plus fondamental pour l'organisme biologique, ce sont sont des valeurs, ou plutôt des rapports de valeur, qui définissent le vivant en tant que vivant.

La citation suivante, tiré d'un essai intitulé « Le Vivant et son milieu » et datant des années cinquante, explique plus clairement cette perspective canghuilhemiste :

5 Gayon, J. (1998) : « The Concept of Individuality in Canguilhem's Philosophy of Biology », Journal of the History of Biology, 31, 3, pp. 305-325, p. 306.

6 Ibid., p. 317. 
Du point de vue biologique, il faut comprendre qu'entre l'organisme et l'environnement, il y a le même rapport qu'entre les parties et le tout à l'intérieur de l'organisme lui-même. L'individualité du vivant ne cesse pas à ses frontières ectodermiques, pas plus qu'elle ne commence à la cellule. Le rapport biologique entre l'être et son milieu est un rapport fonctionnel, et par conséquent mobile, dont les termes échangent successivement leur rôle. La cellule est un milieu pour les éléments infra-cellulaires, elle vit elle-même dans un milieu intérieur qui est aux dimensions tantôt de l'organe et tantôt de l'organisme, lequel organisme vit lui-même dans un milieu qui lui est en quelque façon ce que l'organisme est à ses composants ${ }^{7}$.

Canguilhem nous présente ici un modèle de l'individuation biologique selon lequel il n'existe que des rapports, c'est-à-dire des rapports parmi des rapports et ainsi de suite sans qu'il y ait un fond de substance qui sous-tende la structure relationnelle ainsi posée. Donc il y a des processus biochimiques divers et leurs rapports aux microstructures moléculaires qui forment à leur tour des structures intracellulaires. De telles structures intracellulaires fonctionnent par rapport aux cellules, celles des cellules par rapport aux organes, celles des organes par rapport à celles de l'organisme, et les structures de l'organisme fonctionnent par rapport à l'environnement qui l'entoure. Mais l'essentiel ici c'est que l'organisme à tous ses niveaux d'organisation est constitué par ces rapports fonctionnels. Il ne s'agit pas de rapports entre les éléments ou des substances déjà existants mais plutôt des éléments qui n'existent que dans ces rapports ou grâce au système de référence constitué à partir de ces rapports.

Le rapport chez Canguilhem est toujours un " rapport à »; une relation entre un point spécifique dans une structure qui, se mettant en relation, constitue un vivant en tant que tel dans son intériorité et dans son extériorité. Mais, et c'est ici le point décisif, ce « rapport à » est toujours, en tant que rapport fonctionnel, un rapport de « sens ». Le système de rapports fonctionnels qui constituent l'organisme vivant à tous ses niveaux est toujours un système qui fait sens, ou bien plus précisément qui est un sens. Canguilhem définit « sens » ici dans les termes suivants : «Un sens, du point de vue biologique et psychologique, c'est une appréciation de valeurs en rapport avec un besoin. Et un besoin c'est pour qui l'éprouve et le vit un système de référence irréductible et par là absolu ${ }^{8}$. Donc les rapports biologiques fonctionnels en tant que " rapports à » constituent l'organisme biologique comme un centre de référence de ces rapports et comme un « sens ». Et le sens dans ce contexte doit être compris axiologiquement comme un système de référence qui est vécu d'une façon qui individue l'organisme comme une valeur autonome et discrète. C'est dans ce contexte que Canguilhem constate ou affirme que « L'être de l'organisme c'est son sens $»^{9}$.

Et c'est ici qu'on peut revenir à la question de l'affectivité, du sentir et du ressentir et à la possibilité du toucher qui sous-tend toute affection, affect ou émotion. Car si pour Canguilhem le sens a une portée ontologique dans sa compréhension du vivant, et si le sens est toujours un " rapport à » constitutif de l'organisme en tant que tel, il est également possible de comprendre ce « rapport à » du sens comme un toucher, comme une

\footnotetext{
Canguilhem, G. (1965) : «Le Vivant et son milieu », La Connaissance de la vie. Paris : Vrin, p. 144.

Ibid., p. 154.

Ibid., p. 147.
} 
affectivité fondamentale opérant à tous les niveaux de l'organisation biologique. C'està-dire que l'on peut comprendre les processus biochimiques les plus élémentaires, les interactions des cellules ainsi que des organes du corps vivant et ses interactions sensibles et intelligibles avec l'environnement comme une économie du toucher ou de l'affectivité. Donc le sens et son " rapport à ", considérés comme toucher et affectivité fondamentale, articulent toute la vie biologique, c'est-à-dire toute la structuration dite «interne » de l'organisme aussi bien que son appréhension du monde qui l'entoure et ses interactions avec ce dernier. De ceci on peut tirer la conjecture que ce qu'on nomme affects ou émotions n'est qu'une continuation ou une prolongation de ce toucher ou de cette affectivité fondamentale qui constituent le vivant.

Je ne dis pas, évidemment, que les amibes, des virus ou des bactéries aient ou ressentent des émotions. Mais cela dit je suis persuadé que les affects et les émotions, étant une continuation ou une prolongation d'une affectivité propre aux processus biochimiques les plus élémentaires, ne sont pas foncièrement différents du sentir et du ressentir éprouvés par des organismes biologiques les plus simples. Il ne s'agit que d'une différence de degré et de complexité relationnelle. De là on peut avancer l'hypothèse, non moins spéculative, que la conscience aussi est une continuation ou une prolongation de cette affectivité biologique fondamentale. Le fait de sentir, de ressentir et de ressentir qu'on ressent, serait dans ce contexte la condition de toute visée intentionnelle de la conscience. On approuverait donc tout à fait Spinoza en affirmant que ce sont des affects et l'affectivité en général qui constituent, à sa base, l'Esprit ou la vie consciente.

Mais il n'y a plus ici de parallélisme entre le physique et le psychique. Cette optique qui considère les affects, les émotions et la conscience comme une continuation ou une prolongation des " rapports à " du sens qui organisent la vie biologique en tant que telle, exige une reconfiguration des lignes de partage qui ont séparé l’expérience qualitative et la détermination quantitative. Car l'expérience qualitative ou le vécu phénoménal d'une part et les éléments biologiques déterminés quantitativement de l'autre ne sont plus considérés en parallèle mais en continuité directe. On a proposé sur un ton quelque peu polémique au début de cette réflexion que le parallélisme psychophysique de Spinoza donnait, malgré sa métaphysique moniste, une définition des affects qui s'orientait selon l'expérience qualitative et selon la perspective de la première personne. On a proposé aussi que cette forte orientation vers la dimension qualitative de l'existence immanente se prolongeait dans la métaphysique deleuzienne. À partir de la pensée canguilhemiste, et à considérer les structures dites "physiques » et le sentir ou le ressentir supposé "psychique » également comme des « rapports à » du sens, on arrive à ne plus séparer le quantitatif et le qualitatif, la détermination objective et le vécu phénoménal subjectif.

Et c'est ici, pour conclure, qu'il faut se référer au penseur contemporain prééminent du sens, du toucher et donc de l'affection fondamentale de l'exister en général, c'est-à-dire Jean-Luc Nancy. Car Nancy, tout comme Canguilhem, confère au terme sens une portée ontologique quoique dans une perspective plus large qui excède la biologie. Rappelons que dans Le Sens du monde Nancy souligne lui aussi le caractère relationnel du sens : " Il n'y a de sens que dans un rapport à quelque "dehors" ou "ailleurs" à quoi le sens consiste à se rapporter ${ }^{10}$. Et tout comme Canguilhem décrète, en insistant là-dessus, que l'orga-

10 Nancy, J.-L. (1993) : Le Sens du monde. Paris : Galilée, p. 17. 
nisme biologique est son sens, Nancy pour sa part est convaincu que les rapports et le partage du sens qui constituent les entités ou les singularités existantes font ou plutôt sont le monde : «Ainsi, monde n'est pas seulement corrélatif de sens, il est structuré comme sens, et réciproquement, sens est structuré comme monde » ${ }^{11}$. Ou bien aussi: «le monde n'a plus de sens, mais il est le sens ${ }^{12}$. Cette référence à Nancy ouvre la possibilité de poser les rapports physiques, géophysiques, biophysiques et psychophysiques dans une relation de continuité ontologique les uns avec les autres. En croisant la pensée biologique canguilhemiste et la pensée nancéenne du sens on arrive à une perspective naturaliste qui situe la vie psychique des êtres vivants, leurs sensations, leurs affects, leurs émotions, tout aussi bien que leur conscience du monde et d'eux-mêmes, dans une continuité directe avec la vie physique. Un tel naturalisme est développé plus longuement ailleurs ${ }^{13}$.

La reconfiguration ici proposée des lignes de partage qui séparent le psychique et le physique, le corps biologique et le corps affectif, n'est pas sans conséquence pour d'autres configurations de lignes et de limites. Ne pas séparer la dimension qualitative de l'existence de sa détermination quantitative a également des incidences sur notre façon de distinguer entre les domaines de savoir ou de connaissance, par exemple le rapport de la philosophie ou des sciences humaines aux sciences naturelles dites " dures ». Et ne pas séparer les rapports de sens qui organisent physiquement l'organisme biologique et qui en même temps donnent la possibilité psychique de sentir, de ressentir, d'avoir des émotions et finalement de penser, ceci nous force à repenser la distinction entre la vie animale et la vie humaine. Car, si l'on sait, depuis Darwin au moins, que la vie humaine est dans une continuité biologique avec la vie animale, cette pensée de l'affectivité, du sens et des affects nous montre aussi clairement que les émotions, en tant qu'articulation de la vie biologique et base de la conscience, sont des qualités fondamentales de l'expérience sensible et pour les êtres humains et pour les animaux. Du point de vue qualitatif il n'y a rien d'essentiel à distinguer entre les deux. De la vie affective des organismes élémentaires jusqu’à la vie émotionnelle riche et complexe des mammifères (êtres humains compris), des oiseaux et, qui sait, des reptiles, il ne s'agit que des différences de degré, des différences de complexité et d'articulation relationnelles.

\section{BIBLIOGRAPHIE}

Ansell-Pearson, K. (1997) : Viroid Life: Perspectives on Nietzsche and the Transhuman Condition. London : Routledge.

Ansell-Pearson, K. (1999) : Germinal Life: The Difference and Repetition of Deleuze. London: Routledge. Canguilhem, G. (1965) : « Le Vivant et son milieu », La Connaissance de la vie. Paris : Vrin, p. 144. Churchland, P. (1996) : « The Rediscovery of Light», The Journal of Philosophy, 93, 5, pp. 211-228.

DeLanda, M. (2002) : Intensive Science and Virtual Philosophy. London : Bloomsbury.

Deleuze, G. (1962) : Nietzsche et la philosophie. Paris : Presses Universitaires de France.

Deleuze, G. (1968) : Différence et répétition. Paris : Presses Universitaires de France.

11 Ibid., p. 18.

12 Ibid., p. 19.

13 James, I. (sous presse) : The Technique of Thought: Nancy, Laruelle, Malabou, and Stiegler After Naturalism. Minneapolis : Minnesota University Press. 
Gayon, J. (1998) : « The Concept of Individuality in Canguilhem's Philosophy of Biology », Journal of the History of Biology, 31, 3, pp. 305-325.

James, I. (sous presse) : The Technique of Thought: Nancy, Laruelle, Malabou, and Stiegler After Naturalism. Minneapolis : Minnesota University Press.

Nancy, J.-L. (1993) : Le Sens du monde. Paris : Galilée.

Protevi, J. (2013) : Life, War, Earth: Deleuze and the Sciences. Minneapolis : University of Minnesota Press. Spinoza, B. (1988) : Ethique. Traduit par Bernard Pautrat. Paris : Seuil.

\section{James, Ian}

Faculty of Modern and Medieval Languages

University of Cambridge

Downing College, Cambridge, CB2 1DQ

irj20@cam.ac.uk 